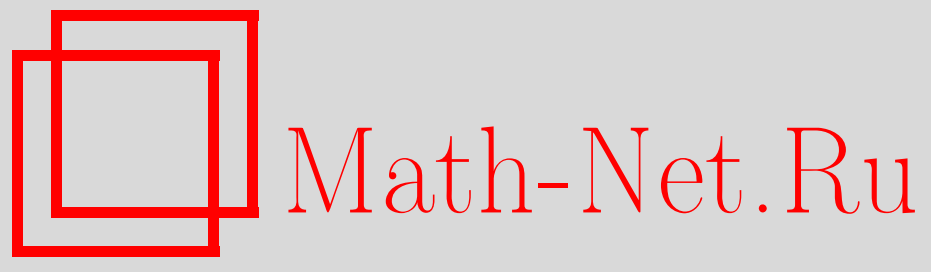

А. А. Муравлёв, Методы последовательного различения гипотез о значении сноса фрактального броуновского движения, УМН, 2013, том 68, выпуск 3, 193-194

DOI: https://doi.org/10.4213/rm9534

Использование Общероссийского математического портала Math-Net.Ru подразумевает, что вы прочитали и согласны с пользовательским соглашением http://www.mathnet.ru/rus/agreement

Параметры загрузки:

IP : 54.89 .56 .158

26 апреля 2023 г., $14: 36: 26$

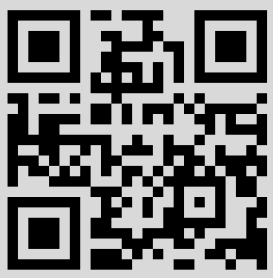




\section{Методы последовательного различения гипотез о значении сноса фрактального броуновского движения}

\section{А. А. Муравлёв}

1. Пусть на вероятностном пространстве $(\Omega, \mathscr{F}, \mathrm{P})$ задан процесс $X=\left(X_{t}\right)_{t \geqslant 0}$,

$$
X_{t}=\mu t+B_{t}^{H},
$$

где $B^{H}=\left(B_{t}^{H}\right)_{t \geqslant 0}$ - фрактальное броуновское движение с параметром Харста $H \in$ $(0,1)$, а $\mu$ - независимая от $B^{H}$ случайная величина (далее мы будем предполагать, что $\mathrm{E}|\mu|<\infty)$. Напомним, что $B^{H}$ определяется как гауссовский процесс с

$$
B_{0}^{H}=0, \quad \mathrm{E} B_{t}^{H}=0, \quad \mathrm{E} B_{s}^{H} B_{t}^{H}=\frac{1}{2}\left(t^{2 H}+s^{2 H}-|t-s|^{2 H}\right), \quad s, t \geqslant 0 .
$$

Процесс $B^{H}$ при $H=1 / 2$ является стандартным броуновским движением, но при $H \neq 1 / 2$ не является ни семимартингалом, ни марковским процессом (см., например, [1]).

2. Пусть множества $A_{1}, \ldots, A_{n} \subset \mathbb{R}$ образуют полную группу, т. е. не пересекаются и $\sum_{i} \mathrm{P}\left(\mu \in A_{i}\right)=1$. Мы рассмотрим задачу различения гипотез $H_{1}, \ldots, H_{n}$, $H_{i}: \mu \in A_{i}$, по последовательным наблюдениям за $X$. Каждая процедура проверки задается решающим правилом $\delta=\left(\tau, d_{\mu}\right)$, состоящим из момента остановки $\tau$ фильтрации $\left(\mathscr{F}_{t}^{X}\right)_{t \geqslant 0}, \mathscr{F}_{t}^{X}=\sigma\left(X_{s} ; s \leqslant t\right)$, и $\mathscr{F}_{\tau}^{X}$-измеримой функции $d_{\mu}$, принимающей значения $1, \ldots, n$. Момент $\tau$ соответствует времени прекращения наблюдения, а значение $d_{\mu}$ - номеру принимаемой гипотезы.

Каждому решающему правилу $\delta$ сопоставим функиию потерь $\mathscr{R}_{X}(\delta)=\mathrm{E}[c \tau+$ $\left.W\left(\mu, d_{\mu}\right)\right]$, где $c \tau$ является платой за наблюдения (пропорциональной времени наблюдения), а $W\left(\mu, d_{\mu}\right)$ - функиией штрафа (за неправильно принятое решение). Рассмотрим задачу отыскания оптимального правила $\delta^{*}=\left(\tau^{*}, d_{\mu}^{*}\right)$ такого, что

$$
\mathscr{R}_{X}\left(\delta^{*}\right)=\inf _{\delta} \mathscr{R}_{X}(\delta),
$$

где инфимум берется по всем решающим правилам $\delta=\left(\tau, d_{\mu}\right)$ с $\mathrm{E} \tau<\infty$. В работе [2] показано, что когда функция штрафа является квадратичной или дельта-функцией, а $\mu \sim \mathscr{N}\left(\mu_{0}, \sigma_{0}^{2}\right)$, оптимальный момент $\tau^{*}$ (в классе правил $\delta$ с $\tau \leqslant T, T>0$ ) является детерминистическим. В (1) момент $\tau^{*}$, вообще говоря, является случайным.

3. Рассмотрим интегральный оператор $M$, преобразующий $f$ в $M(f)$ согласно

$$
M_{t}(f)=c_{H} \int_{0}^{t} s^{1 / 2-H}(t-s)^{1 / 2-H} d f_{s}, \quad c_{H}=\left(\frac{\Gamma(3-2 H)}{2 H \Gamma(3 / 2-H)^{3} \Gamma(1 / 2+H)}\right)^{1 / 2} .
$$

Определим процесс $Z=\left(Z_{t}\right)_{t \geqslant 0}$ как $Z_{t}=M(X)_{\langle M(X)\rangle_{t}^{-1}}$, где $\langle M(X)\rangle_{t}=t^{2-2 H}$ есть квадратическая характеристика $M(X)$.

Теорема 1. Прочесс $Z$ допускает представление $Z_{t}=\lambda t+B_{t}$, где $B=\left(B_{t}\right)_{t \geqslant 0}$ есть стандартное броуновское движение, а $\lambda$ - независимая от $B$ случайная величина. При этом фильтрации $\left(\mathscr{F}_{t}^{X}\right)_{t \geqslant 0} u\left(\mathscr{F}_{t}^{Z}\right)_{t \geqslant 0}$ совпадают.

Доказательство основано на том, что процесс $M\left(B^{H}\right)$ является (фундаментальным) мартингалом, причем фильтрации $B^{H}$ и $M\left(B^{H}\right)$ совпадают (см., например, [1], [3]). В качестве $B_{t}$ выбирается $M\left(B^{H}\right)_{\left\langle M\left(B^{H}\right)\right\rangle_{t}^{-1}}$, а $\lambda=b_{H} \mu$, где $b_{H}=c_{H} \mathrm{~B}(3 / 2-H, 3 / 2-H)$.

Работа выполнена при поддержке "Премолаб", МФТИ (грант правительства РФ, дог. 11.G34.31.0073) и РФФИ (гранты № 11-01-00949-a, 12-01-31449-мол_а, 13-08-01096-а).

DOI: $10.4213 / \mathrm{rm} 9534$ 
Каждому $\left(\mathscr{F}_{t}^{Z}\right)_{t \geqslant 0}$-решающему правилу $\gamma=\left(\sigma, d_{\lambda}\right)$ сопоставим $\left(\mathscr{F}_{t}^{X}\right)_{t \geqslant 0 \text {-решающее }}$ правило $\delta=\left(\tau, d_{\mu}\right)$ с $\tau=\sigma^{1 /(2-2 H)}, d_{\mu}(\tau) \equiv d_{\lambda}(\sigma)$. Несложно видеть, что это отображение (назовем его $\mathscr{A}$ ) является биекиией. Рассмотрим задачу

$$
\mathscr{R}_{Z}\left(\gamma^{*}\right)=\inf _{\gamma} \mathscr{R}_{Z}(\gamma), \quad \mathscr{R}_{Z}(\gamma)=\mathrm{E}\left[c \sigma^{1 /(2-2 H)}+\widetilde{W}\left(\lambda, d_{\lambda}\right)\right],
$$

где $\widetilde{W}\left(\lambda, d_{\lambda}\right)=W\left(\mu, d_{\mu}\right)$ и инфимум берется по всем $\gamma=\left(\sigma, d_{\lambda}\right)$ с $\mathrm{E} \sigma^{1 /(2-2 H)}<\infty$.

Теорема 2. Решения задач (1) u (2) совпадают, m.е. $\mathscr{R}_{X}\left(\delta^{*}\right)=\mathscr{R}_{Z}\left(\gamma^{*}\right)$, при этом оптимальные правила связаны соотношением $\delta^{*}=\mathscr{A} \gamma^{*}$.

4. Следуя общей теории статистического последовательного анализа [4], получаем

$$
\mathscr{R}_{Z}\left(\gamma^{*}\right)=\inf _{\sigma} \mathrm{E}\left[c \sigma^{1 /(2-2 H)}+\min _{i} \mathrm{E}\left(\widetilde{W}(\lambda, i) \mid \mathscr{F}_{\sigma}^{Z}\right)\right],
$$

где инфимум берется по моментам остановки $\sigma$ таким, что $\mathrm{E}^{1 /(2-2 H)}<\infty$. При этом, если $\sigma^{*}$ является оптимальным, то правило $\delta^{*}=\left(\sigma^{*}, d_{\lambda}^{*}\right)$ с $d_{\lambda}^{*}=\arg \min _{i} \mathrm{E}\left(\widetilde{W}(\lambda, i) \mid \mathscr{F}_{\sigma^{*}}^{Z}\right)$ является оптимальным в (2). Пусть $\mathrm{P}^{0}$ обозначает меру $\mathrm{P}^{0}(\cdot)=\mathrm{P}(\cdot \mid \lambda=0)$, по которой процесс $Z$ является броуновским движением. Рассмотрим задачу

$$
V=\inf _{\sigma} \mathrm{E}^{0}\left[c \sigma^{1 /(2-2 H)} \mathrm{E}^{0}\left(\mathscr{E}_{\sigma}(\lambda) \mid \mathscr{F}_{\sigma}^{Z}\right)+\min _{i} \mathrm{E}^{0}\left(\widetilde{W}(\lambda, i) \mathscr{E}_{\sigma}(\lambda) \mid \mathscr{F}_{\sigma}^{Z}\right)\right],
$$

где $\mathscr{E}_{\sigma}(\lambda)=\exp \left(\lambda Z_{\sigma}-\lambda \sigma^{2} / 2\right), \mathrm{E}^{0}-$ математическое ожидание по мере $\mathrm{P}^{0}$, а инфимум берется по всем моментам $\sigma$ таким, что $\mathrm{E}^{0} \sigma^{1 /(2-2 H)}<\infty$.

Теорема 3. Пусть $\sigma^{*}$ - оптимальный момент в задаче (4) $и \mathrm{P}\left(\sigma^{*}<\infty\right)=1$. Тогда $\sigma^{*}$ является оптимальным также и в задаче (3) $и \mathscr{R}_{Z}\left(\gamma^{*}\right)=V$.

Доказательство основано на общей теории абсолютной непрерывности мер процессов Ито относительно меры винеровского процесса (см., например, [5; ч. 7]). Чтобы перейти к задаче для броуновского движения в случае $\mu \sim \mathscr{N}\left(\mu_{0}, \sigma_{0}^{2}\right)$, вместо замены меры можно использовать гауссовость $Z$ по Р и метод замены времени (см. [6]).

5. Итак, мы перешли от задачи (1) к (4). Отметим, что (4) является стандартной задачей, поскольку имеет вид $V=\inf _{\sigma} \mathrm{E}^{0} G\left(\sigma, Z_{\sigma}\right)$. Для ее изучения можно использовать хорошо разработанные методы теории оптимальной остановки броуновского движения (см., например, [7], [8]).

Примеры решения конкретных задач будут рассмотрены в другой статье.

Автор выражает признательность А.Н. Ширяеву за внимание к работе.

\section{Список литературы}

[1] Yu. S. Mishura, Stochastic calculus for fractional Brownian motion and related processes, Lecture Notes in Math., 1929, Springer, Berlin, 2008, xviii+393 pp. [2] U. Çetin, A. Novikov, A. N. Shiryaev, "Bayesian sequential estimation of a drift of fractional Brownian motion", Sequential Anal., 2013 (to appear). [3] Г. М. Молчан, Теория веролтн. и ее примен., 14:3 (1969), 556-559. [4] А.Н. Ширяев, Статистический последовательный анализ. Оптимальные правила остановки, Наука, М., 1969, 231 с. [5] Р.Ш. Липцер, А. Н. Ширяев, Статистика случайных прочессов: нелинейная фильтрачия и смежные вопросы, 15, Наука, М., 1974, 696 с. [6] М. В. Житлухин, А.А. Муравлёв, Теория вероятн. и ее примен., 57:4 (2012), 778-788. [7] G. Peskir, A. Shiryaev, Optimal stopping and free-boundary problems, Birkhäuser, Basel, 2006, xxii+500 pp. [8] P. van Moerbeke, Arch. Ration. Mech. Anal., 60:2 (1976), 101-148.

А. А. Муравлёв (А. А. Muravlev)

Математический институт им. В. А. Стеклова РАН

E-mail: almurav@mi.ras.ru
Представлено А. В. Булинским Принято редколлегией 05.04.2013 